\title{
Associations between Milk and Egg Allergens and the HLA-DRB1/DQ Polymorphism: A Bioinformatics Approach
}

\author{
Ivan Dimitrov Irini Doytchinova \\ Faculty of Pharmacy, Medical University of Sofia, Sofia, Bulgaria
}

\section{Key Words}

Food allergy · Egg white allergens · Egg yolk allergens .

Milk allergens $\cdot$ HLA-DRB1 $\cdot$ HLA-DQ

\begin{abstract}
Background: Little is known about the associations between human leukocyte antigens (HLAs) and food allergies. Our aim was to analyze the associations between the HLA class II polymorphism and food allergy using bioinformatics. Methods: A two-step algorithm was developed which mimics the food allergen processing in the human body. In the first step, the allergen is digested by pepsin, trypsin and chymotrypsin. In the second step, the digested fragments bind to the most frequent 12 HLA-DRB1 and 5 HLA-DQ alleles, and the binding affinities are predicted. Results: The algorithm was applied to 13 well-known milk and egg allergens. The predicted HLA binders were compared to known T-cell and IgE epitopes originating from the same allergens, and $77 \%$ of them were found to overlap. We found that the peptides generated from milk allergens bind to DRB1*01:01, DQ7 and DQ8 but not to DRB1*03:01, DRB1*04:04, DRB1*12:01 and $\mathrm{DRB} 1 * 15: 01$. The peptides generated from egg allergens bind to $\mathrm{DRB} 1{ }^{*} 01: 01, \mathrm{DQ} 4, \mathrm{DQ} 7$ and $\mathrm{DQ} 8$ but not to $\mathrm{DRB} 1{ }^{*} 03$ : 01, DRB1*04:04 and DRB1*12:01. They bind to all the DQs studied. The alleles that bind to allergen peptides could be considered as susceptible to the particular allergy and the
\end{abstract}

\section{KARGER}

E-Mail karger@karger.com

www.karger.com/iaa nonbinding alleles as protective. Conclusions: The alleles DRB1*01:01, DQ7 and DQ8 are considered as susceptible to cow's milk allergy and DRB $1 * 03: 01$, DRB $1 * 04: 04, \mathrm{DRB} 1 * 12: 01$ and DRB $1 * 15: 01$ as protective. The alleles DRB $1 * 01: 01$, DQ4, DQ7 and DQ8 are considered as susceptible to egg allergy and DRB1*03:01, DRB1*04:04 and DRB1*12:01 as protective. Protective DQs against egg allergy were not revealed in this study.

(c) 2016 S. Karger AG, Basel

\section{Introduction}

The major histocompatibility complex (MHC) class II proteins are involved in allergen processing in the specialized antigen-presenting cells located at mucosal surfaces. In humans, the MHC proteins are known as human leukocyte antigen (HLA) proteins. The HLA class II proteins involved in the presentation of allergen fragments to the Th2 cells are extremely polymorphic and polygenic [1]. They consist of 2 protein chains, $\alpha$ and $\beta$, encoded by 3 HLA loci: DR, DQ and DP. The binding cleft on HLA class II proteins is occupied by nonamer peptides but is open-ended, allowing much longer peptides than nonamers to bind. The HLA polymorphism is concentrated in the binding cleft. Each cleft consists of several binding pockets which have a different shape
(C) 2016 S. Karger AG, Basel

$1018-2438 / 16 / 1691-0033 \$ 39.50 / 0$
Correspondence to: Dr. Irini Doytchinova

Faculty of Pharmacy, Medical University of Sofia 2 Dunav Street

BG-1000 Sofia (Bulgaria)

E-Mail idoytchinova@pharmfac.net 
and polarity, and hence the ability to bind different amino acid residues [2-4].

The HLA class II polymorphism is associated with susceptibility to and protection against some allergies. Knapp et al. [5] found an association between HLA-DR1 and the mugwort pollen allergen, Art v 1. Rajagopalan et al. [6] reported that HLA-DR3 is associated with childhood asthma, while other studies found no association between atopic asthma and HLA [7, 8]. Kusano et al. [9] analyzed the association between HLA-DP5 and the Japanese cedar pollen allergen, Cry $\mathrm{j} 1$. Associations between several DR and DQ alleles and the cow dander allergen, Bos d 2, have been reported [10]. The known HLA class II restrictions of allergens are reviewed by Jahn-Schmid et al. [11].

As peanut allergy is the most common, severe, usually permanent and increasingly prevalent food allergy, and is also associated with substantial morbidity and mortality [12], most studies looking for associations between the HLA polymorphism and food allergy focus on peanut allergy [13-17]. Less is known about the association between the HLA polymorphism and milk allergy. It was found that the sequence in $\alpha_{\mathrm{S} 1}$-casein most immunogenic to T cells from children with cow's milk allergy (CMA) contained T-cell epitopes restricted to DQB1*0201, DPB1*0401 and DRB1*1501 [18]. In another study, the DR15-DQB1*06:02 haplotype was associated with high levels of $\beta$-lactoglobulin-specific and $\alpha$-casein-specific IgG, while the DR1/10-DQB1*05:01 haplotype was associated with lower levels of $\beta$-lactoglobulin-specific IgG [19]. In the same study, one association between HLA polymorphism and egg allergy was found: the DR1/10-DQB1*05:01 haplotype was associated with lower levels of ovalbumin-specific IgG.

In this study, we apply a bioinformatics approach to analyze the associations between the 12 most frequent HLA-DRB1 and 5 most frequent HLA-DQ alleles and 13 known milk and egg allergens. The in silico processing mimicked the allergen processing in the human body. Initially, food allergens were degraded by 3 digestive enzymes, trypsin, chymotrypsin and pepsin. The digested fragments then bound to DR and/or DQ molecules with a different affinity. The stable peptide/HLA complexes were presented at the cell surface for Th cell inspection. The final set of peptides was compared to known allergen fragments (epitopes). Several associations between some HLA class II alleles and tested allergens were detected.

\section{Datasets and Methods}

HLA Class II Proteins Used in the Study

The 12 most frequent HLA-DRB1 and 5 most frequent HLADQ alleles were used in the study [20]. The DRB1s are: ${ }^{*} 01: 01,{ }^{*} 03$ : 01, *04:01, *04:04, *04:05, *07:01, *08:02, *09:01, *11:01, *12:01, *13:02 and ${ }^{*} 15: 01$. The DQs are: DQ4 (DQA ${ }^{*} 04: 01 / \mathrm{DQB} 1 * 04:$ 02), DQ5 (DQA1*01:01/ DQB1*05:01), DQ6 (DQA1*01:02/ DQB1*06:02), DQ7 (DQA1*05:01/ DQB1*03:01) and DQ8 (DQA1*03:01/DQB1*03:02).

\section{Milk and Egg Allergens Used in the Study}

The allergenic proteins from milk and egg were selected from the Allergome database [21] and checked for existence on protein level versus UniProt knowledgebase [22]. Thirteen known allergenic proteins from milk, egg white and egg yolk were used in the study. The milk proteins are: $\alpha$-lactalbumin (Bos d 4), $\beta$-lactoglobulin (Bos d 5), bovine serum albumin (Bos d 6), $\alpha_{S 1^{-}}$ casein (Bos d 9), $\alpha_{S 2}$-casein (Bos d 10), $\beta$-casein (Bos d 11) and $\kappa$-casein (Bos d 12). The egg white proteins are: ovomucoid (Gal d 1), ovalbumin (Gal d 2), ovotransferrin (Gal d 3) and lysozym C (Gal d 4). The egg yolk proteins are $\alpha$-livetin (Gal d 5) and vitellogenin (Gal d 6). The full-length protein sequences were downloaded from UniProt in FASTA format.

Both milk and egg are normally consumed after thermal processing. Whey milk proteins (Bos d 4, Bos d 5 and Bos $\mathrm{d} 6$ ) denature progressively upon heat treatment [23]. However, the caseins (Bos d 9, Bos d 10, Bos d 11 and Bos d 12) are heat-stable because they lack secondary, tertiary and quaternary structures that can be destroyed by heating. Thus, heating milk can only partly reduce its allergenicity [23].

Egg is one of the foods which has its allergenicity most altered by heating [24]. The thermal stability of egg allergens ranges from the heat-resistant Gal d 1, Gal d 5 and Gal d 6 to the partially heatlabile Gal d 4 and the heat-sensitive Gal d 2 and Gal d 3.

Despite the thermal instability of some milk and egg allergens, all of them were considered in this study.

Known T-Cell and IgE Epitopes from Milk and Egg Allergens

Information about T-cell epitopes was available for only 4 milk allergens, Bos d 4, Bos d 5, Bos d 6 and Bos d 9, and for 2 egg allergens, Gal d 1 and Gal d 2. The information contained neither $\mathrm{IC}_{50}$ values nor HLA restriction.

Information about IgE epitopes originating from the studied allergenic proteins was available for 7 milk allergens, Bos d 4, Bos d 5, Bos d 6, Bos d 9, Bos d 10, Bos d 11 and Bos d 12, and for 3 egg allergens, Gal d 1, Gal d 2 and Gal d 4 . In the case of multiple overlapping epitopes, the longest one was selected.

\section{PeptideCutter: Server for in silico Protein Digestion}

The server PeptideCutter predicts potential cleavage sites cleaved by proteases or chemicals in a given protein sequence [25]. The server accepts the test protein as a 1-letter amino acid sequence or in FASTA format. The user selects the proteolitic enzymes from a wide range of enzymes. Our study used the enzymes trypsin and chymotrypsin with high and low specificity, pepsin at $\mathrm{pH} 1.3$ and pepsin at $\mathrm{pH}>2$. The server returns the query sequence with the possible cleavage sites mapped on it and/or a table of cleavage site positions. After the in silico digestion, each protein is presented as a set of peptides with different lengths and even single 


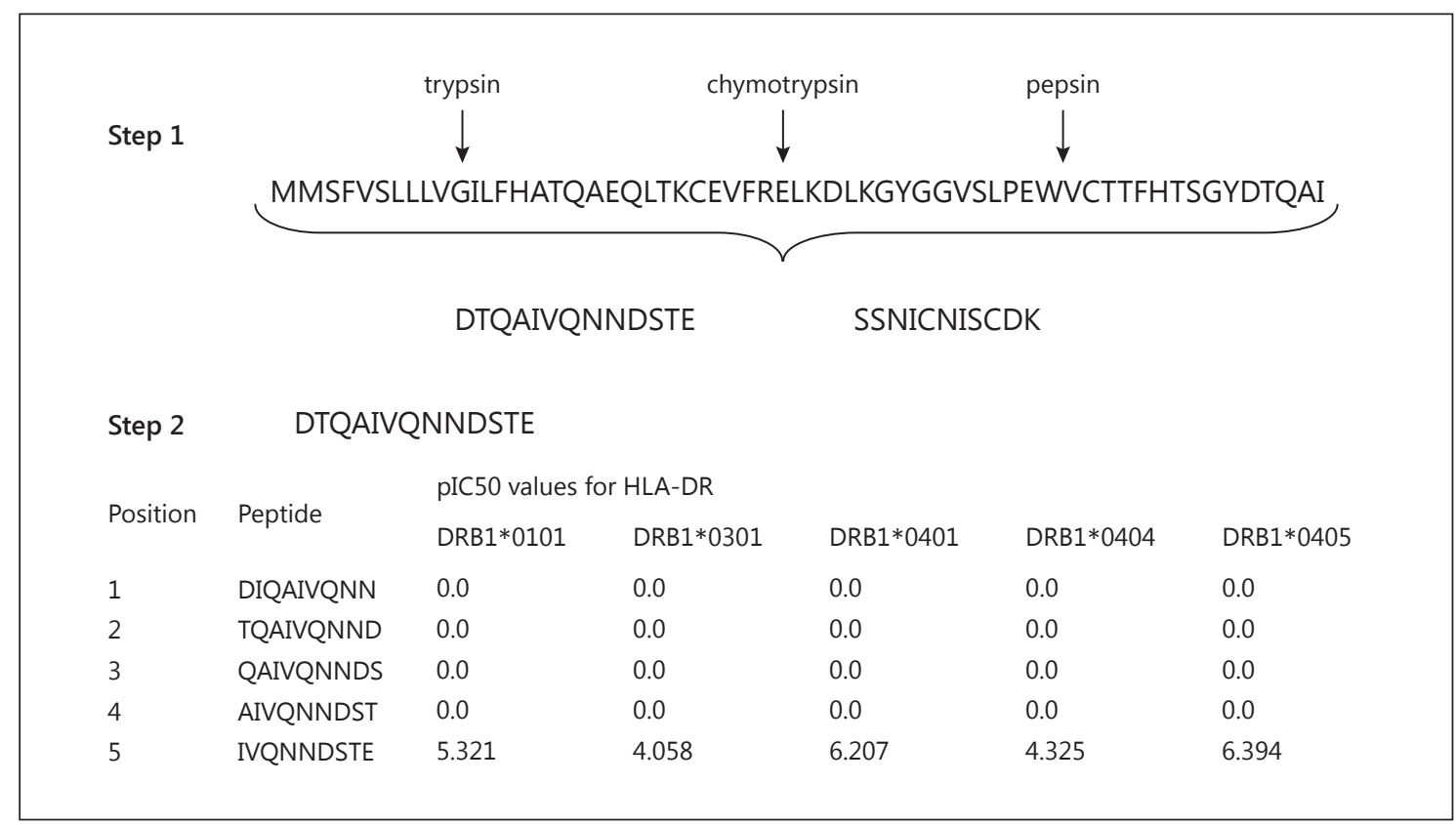

Fig. 1. Two-step algorithm for in silico food allergen processing in the human body. At the first step, the allergen is digested by pepsin, trypsin and chymotrypsin. At the second step, the binding affinities of the digested fragments to the most frequent HLA-DRB1 and HLA-DQ alleles are predicted.

amino acids. As the peptide-binding groove of MHC class II proteins accepts 9 residues, only peptides equal to or longer than 9 amino acids are selected and used in the next step, i.e. HLA class II binding prediction.

EpiTOP: Server for HLA Class II Binding Prediction

EpiTOP is a server for HLA class II binding prediction using proteochemometric models $[26,27]$. It is a web-based application written in Python and HTML and integrating the MySQL database environment. EpiTOP v2 identifies peptides binding to 12 HLADRB1 and 5 HLA-DQ proteins within protein sequences, with options to vary HLA allele and cut-off. EpiTOP v2 is freely accessible at http://www.ddg-pharmfac.net/epitop.

\section{Results}

In order to analyze the associations between the DRB1 and DQ alleles and the known milk and egg allergens, the allergen processing in the human body was mimicked in silico. The algorithm is presented in figure 1. Initially, the food allergen digestion in the gastrointestinal tract was predicted by PeptideCutter. Three main digestive enzymes were considered: pepsin, trypsin and chymotrypsin. The peptide fragments gained after the digestion were endocytozed by antigen-presenting cells where they are processed and bind to HLA class II proteins with a differ- ent affinity. The $\mathrm{IC}_{50}$ values of the peptide/HLA complexes were predicted by EpiTOP. Only peptides with a $\mathrm{pIC}_{50}$ $>6.3\left(\mathrm{IC}_{50}=500 \mathrm{nM}\right)$ were considered as binders. These peptides were compared to known allergen epitopes.

\section{The in silico Digestion of Allergens}

The ability of food allergens to be stable to digestion by the gastrointestinal tract is one of the prerequisites for allergenicity [28]. The in silico enzymatic treatment cuts the proteins into short peptides. Most of the resultant fragments consist of $<9$ residues. In order to interact with the immune system, the allergenic proteins or a part of them need to survive the digestion and to reach the antigen-presenting cells with an appropriate length. In our study, only peptides equal to or longer than 9 residues were considered. The initial lengths of the studied allergens and the number of peptides generated after the in silico digestion are shown in online supplementary table 1 (see www.karger.com/doi/10.1159/000444172 for all online suppl. material). The effectiveness of the digestion process is presented by percent of reduction: (initial protein length - total lengths of the resulted peptides)/ initial protein length (online suppl. table 1). For example, the allergen Bos $\mathrm{d} 4$ consists of 142 residues. After the digestion, it cleaved into 2 peptides equal to or longer than 
9 residues (online suppl. table 2). The total length of the 2 fragments was 24 residues, i.e. $17 \%$ of the initial length. Therefore, the reduction of Bos $\mathrm{d} 4$ after in silico digestion was $83 \%$. The effect of the in silico enzyme digestion varies from a significant reduction (Bos d 5 and Bos $\mathrm{d} 6$ with 92\%) to a moderate reduction (Bos d 12 with 64\%) with an average value of $81 \%$. The peptides generated after the in silico digestion and their corresponding known epitopes are given in online supplementary table 3 .

The protein fragments generated after the in silico digestion were compared to the sets of known T-cell and/ or IgE epitopes from the same allergens. If a 9-residue overlap between them existed, the predicted epitope was considered as a true positive. Sensitivity is defined as the ratio between true positive epitopes to all protein fragments generated after the in silico digestion. An average sensitivity of $77 \%$ was achieved, i.e. $77 \%$ of the peptides generated after the in silico digestion overlapped with at least 1 known T-cell or IgE epitope.

\section{The in silico DRB1 and DQ Binding Prediction of Allergen Fragments}

The protein fragments generated after the in silico digestion were entered into EpiTOP, where they were chopped into nonamers and their binding affinities to the most frequent DRB1 and DQ proteins were predicted (online suppl. table 2). In general, the nonamers binding to DRB1 are significantly fewer than those binding to DQ (online suppl. tables 4, 5). This is explained by the fact that the most important anchor for DRB1 binding, i.e. the peptide position 1 ( $\mathrm{p} 1$ ), requires hydrophobic residues as Leu, Ile, Phe, Trp, Met, Val, Tyr and Ala [2]. Only peptides bearing such residues at $\mathrm{p} 1$ are able to bind to DRB1 proteins. In contrast, the peptides binding to DQ have quite diverse structures. Most of the surviving peptides were found to bind to the 5 studied DQ proteins.

The average predicted $\mathrm{pIC}_{50}$ values of the milk allergens indicated that Bos $\mathrm{d} 11$ showed the highest affinity to DRB1 with an average $\mathrm{pIC}_{50}$ of $7.140\left(\mathrm{IC}_{50}=72 \mathrm{nM}\right)$, followed by Bos d 6 ( $\left.\mathrm{pIC}_{50}=7.114, \mathrm{IC}_{50}=77 \mathrm{nM}\right)$ and Bos $\mathrm{d} 12\left(\mathrm{pIC}_{50}=6.908, \mathrm{IC}_{50}=124 \mathrm{nM}\right.$; online suppl. table 4$)$. Bos d 12 contains the most DRB1 binders, i.e. 39, while Bos $\mathrm{d} 4$ has only 2. The highest affinity to DQ proteins was shown by Bos d 6 with an average $\mathrm{pIC}_{50}$ of $7.100\left(\mathrm{IC}_{50}=\right.$ $79 \mathrm{nM})$, followed by Bos d $12\left(\mathrm{pIC}_{50}=6.979, \mathrm{IC}_{50}=105\right.$ $\mathrm{nM})$ and Bos d $11\left(\mathrm{pIC}_{50}=6.970, \mathrm{IC}_{50}=107 \mathrm{nM}\right.$; online suppl. table 5). The number of good binders to DQ was significantly higher than the number of binders to DRB1. Bos d 12 contained 101 binders to DQ, while Bos d 6 had only 13.
The egg allergens contained more binders to both DRB1 and DQ proteins, with a slightly lower affinity (online suppl. tables 4, 5). Gal d 2 and Gal d 6 showed the highest affinity to DRB1 ( $\mathrm{pIC}_{50}=7.003$ and 6.977, respectively), while Gal d 4 showed the highest average affinity to $\mathrm{DQs}$ ( $\mathrm{pIC}_{50}=7.605, \mathrm{IC}_{50}=25 \mathrm{nM}$ ) with only 5 good binders. As it has the biggest molecular mass, Gal d 6 contains the largest number of binders.

Milk and egg allergens bound best to DRB1*01:01, with an average $\mathrm{pIC}_{50}$ of 7.513 and 7.198, respectively. For milk allergens, the next most attractive were DRB1*07:01 (average $\mathrm{pIC}_{50}=6.871, \mathrm{IC}_{50}=135 \mathrm{nM}$ ) and $\mathrm{DRB1}{ }^{*} 08: 02$ (average $\left.\mathrm{pIC}_{50}=6.835, \mathrm{IC}_{50}=146 \mathrm{nM}\right)$. The egg allergens preferred DRB1 $* 15: 01$ (average $\mathrm{pIC}_{50}=6.954, \mathrm{IC}_{50}=110$ $\mathrm{nM})$ and DRB1 ${ }^{*} 07: 01$ (average $\mathrm{pIC}_{50}=6.944, \mathrm{IC}_{50}=114$ $\mathrm{nM})$. In terms of the number of binders, DRB $1^{*} 04: 05$, DRB1*01:01, DRB1*07:01 and DRB1*04:01 were preferred by milk and egg proteins over the rest of the DRB1 alleles. No binders were found for DRB $1 * 03: 01, \mathrm{DRB} 1 * 04$ : 04 and DRB $1 * 12: 01$. Only 2 egg binders (but with a high affinity) were found for DRB1*15:01.

Regarding the studied DQ proteins, milk allergens preferred binding to DQ7 (average $\mathrm{pIC}_{50}=7.028, \mathrm{IC}_{50}=$ $94 \mathrm{nM}$ ), DQ8 (average $\mathrm{pIC}_{50}=7.006, \mathrm{IC}_{50}=99 \mathrm{nM}$ ) and DQ4 (average $\mathrm{pIC}_{50}=6.955, \mathrm{IC}_{50}=111 \mathrm{nM}$ ). Ruiter et al. [18] have found that the DQB1*05:01 allele (part of DQ5) frequency is lower in children with CMA than in nonatopic children. The preferences of egg allergens for DQ proteins are in the same order: DQ7 (average $\mathrm{pIC}_{50}=$ $7.176, \mathrm{IC}_{50}=67 \mathrm{nM}$ ), DQ8 (average $\mathrm{pIC}_{50}=7.135, \mathrm{IC}_{50}=$ $73 \mathrm{nM}$ ) and DQ4 (average $\mathrm{pIC}_{50}=7.096, \mathrm{IC}_{50}=80 \mathrm{nM}$ ). The most binders were found for DQ6 and DQ7. Gal d 4 did not bind to DQ5.

\section{Discussion}

In this study, we attempted to analyze the associations between HLA polymorphism and food allergy using bioinformatics. We developed a two-step algorithm mimicking the food allergen processing in the human body. In the first step, the allergen is digested by pepsin, trypsin and chymotrypsin. In the second step, the digested fragments bind to the 12 most frequent HLA-DRB1 and 5 most frequent HLA-DQ alleles and the binding affinities are predicted. We applied this algorithm to 13 known milk and egg allergens. The peptides derived after the in silico digestion were compared to known Tcell and $\operatorname{IgE}$ epitopes originating from the same allergens, and $77 \%$ of them overlapped. It should be mentioned that most of the
Dimitrov/Doytchinova 
known T-cell and IgE epitopes have been derived by tests on overlapping peptide libraries spanning the primary sequences of whole, intact allergenic proteins. No digestion was considered, resulting in an overestimation of the number of both T-cell and IgE epitopes.

Bos d 4 contains several IgE epitopes (online suppl. table 2). Some of them overlap. These epitopes have been found in the intact protein. However, after the in silico digestion, only 2 peptide fragments $\left({ }^{56} \mathrm{D}-\mathrm{E}^{68}\right.$ and $\left.{ }^{88} \mathrm{~S}-\mathrm{K}^{98}\right)$ survived that were longer than 9 amino acids (online suppl. tables 2,3). They belong to the known IgE epitopes ${ }^{36} \mathrm{G}-\mathrm{K}^{77}$ and ${ }^{79} \mathrm{~W}-\mathrm{K}^{98}$, respectively. Bos $\mathrm{d} 5$ is the most abundant whey protein in cow's milk. It has a specific tertiary structure resistant to pepsin digestion and this makes it highly allergenic [29]. The subsequent exposure to duodenal enzymes completely degraded the protein in 30 min [30]. Nevertheless, scanning the sequence of the intact protein by a library of overlapping peptides revealed many IgE and T-cell epitopes (online suppl. table 2). The single peptide found after the in silico digestion, ${ }^{124}$ ENSAEPEQS ${ }^{132}$, is part of the T-cell epitope ${ }^{113} \mathrm{~T}$ $\mathrm{L}^{133}$, but it has no affinity to any of the DRB1 and DQ alleles considered in this study (online suppl. table 3 ). Bos $\mathrm{d} 6$ is the major beef allergen. Testing the regions in which the amino acid sequences of Bos d 6 differ significantly from human serum albumin, Tanabe et al. [31] identified $3 \mathrm{~T}$-cell epitopes and $4 \mathrm{IgE}$ epitopes from the intact protein (online suppl. table 2). However, after the in silico digestion, none of them survived (online suppl. table 3 ).

The casein fraction of bovine milk consists of 4 proteins: $\alpha_{\mathrm{S} 1}$-casein (Bos d 9), $\alpha_{\mathrm{S} 2}$-casein (Bos d 10), $\beta$-casein (Bos d 11) and $\mathrm{k}$-casein (Bos d 12). Caseins are very susceptible to proteases and peptidases and undergo rapid breakdown during digestion [32]. Despite the excellent digestibility of caseins, they act as potent allergens [32, 33 ], which means that sufficient immunologically active fragments survive after the digestion [34]. After the in silico digestion of Bos d 9, 4 peptides were generated (online suppl. table 2). Two of them were present in the fragments derived after the in silico digestion (online suppl. table 3). Twelve IgE epitopes were identified in the intact protein (online suppl. table 1). Most of them are from the same regions as the T-cell epitopes. All 4 digested fragments are parts of the known IgE epitopes (online suppl. table 3). Three peptides were generated after the in silico digestion of Bos d 10. Two of them are present in the known IgE epitopes (online suppl. table 3 ). No T-cell epitopes have yet been identified for this allergen. Bos $\mathrm{d} 11$ is the second most abundant protein in cow's milk with a high content of prolines and a lack of disulfide bonds.
Like other caseins, Bos d 11 is highly sensitive to proteolysis and it is totally digested in the gastrointestinal tract. Five peptides, equal to or longer than 9 amino acids, were generated after the in silico digestion. All of them are part of known IgE epitopes (online suppl. tables 2, 3). No Tcell epitopes have yet been identified for Bos d 11. Bos $d$ 12 differs from other caseins in that it contains disulfide bonds and is more structured. No T-cell epitopes were identified but several IgE epitopes exist in the intact protein (online suppl. tables 2, 3). Four protein fragments remained after the in silico digestion. Two of them belong to the known IgE epitopes.

Gal $\mathrm{d} 1$ is the dominant allergen of hens' egg white. It is relatively stable against heat [35] and proteinases [36]. Seven protein fragments survived after the in silico digestion. Four of them are part of $3 \mathrm{~T}$-cell and $1 \mathrm{IgE}$ epitopes (online suppl. table 3 ). The total number of T-cell epitopes identified was 10, while there were 8 known IgE epitopes (online suppl. table 2). Gal d 2 is one of the major allergens in patients with hen's egg allergy [37]. The IgE epitope mapping for the entire primary sequence of Gal d 2 performed by different authors has identified several epitopes covering almost the whole protein (online suppl. table 2). Five T-cell epitopes were found (online suppl. table 2). Six peptides survived after the in silico digestion of Gal d 2 (online suppl. table 3). One of them is part of a $\mathrm{T}$-cell epitope, and the other 5 belong to the known IgE epitopes. Gal $\mathrm{d} 3$ is an iron-binding protein, and is a heatlabile and digestible allergen [28]. Neither T-cell nor IgE epitopes have been reported for it. Gal d 4 is an enzyme with antimycobacterial activity. It is less allergenic than Gal $\mathrm{d} 1$ and Gal d 2 [38]. Three IgE-binding peptides were identified (online suppl. table 2). The single peptide generated after the in silico digestion overlapped partially with the epitope ${ }^{108} \mathrm{~A}-\mathrm{G}^{122}$ (online suppl. table 3). Gal d 5 and Gal $\mathrm{d} 6$ are hen's egg yolk allergens. Yolk proteins are less allergenic than egg white proteins. Both are heat-resistant and heat treatment does not affect their allergenicity $[39,40]$. The simulated gastric acid digestion completely eliminates the IgE reactivity of Gal d 6 [41]. The in silico digestion generated 5 fragments for Gal d 5 and 33 fragments for Gal d 6 (online suppl. table 2). There are no known $\mathrm{T}$-cell and IgE epitopes for these.

The predicted binding affinities of the protein fragments generated after the in silico digestion of milk and egg allergens suggest several clear associations between the susceptibility to/protection against milk/egg allergy and the HLA-DRB1/DQ polymorphism. The milk allergens showed the highest affinity (average $\mathrm{pIC}_{50}>7, \mathrm{IC}_{50}$ $<100 \mathrm{nM}$ ) to DRB1*01:01, DQ7 and DQ8. These alleles 
could be considered as susceptible to CMA. The milk allergens did not bind to DRB1*03:01, DRB1*04:04, DRB1*12:01 and DRB1*15:01. These alleles could be considered as protective against CMA. The egg allergens showed the highest affinity to DRB1*01:01, DQ4, DQ7 and DQ8 and they did not bind to DRB1*03:01, DRB1*04: 04 and DRB ${ }^{*} 12: 01$. Protective DQ alleles against egg allergy could not be detected, as all of the allergens, except for Bos d 5 and Gal d 4, bound to the DQs studied.

The results that we derived were compared to the sparse information available in the literature regarding the associations between the HLA polymorphism and milk/egg allergen proteins. Ruiter et al. [18] used a set of overlapping peptides, spanning the Bos d 9 molecule to stimulate T-cell clones derived from children with and without CMA and from nonatopic children. They have found that Bos d 9 contains $\mathrm{T}$ cell epitopes restricted to DQB1*02:01, DPB $1 * 04: 01$ and DRB1*15:01. Of the 3 alleles, only DRB1*15:01 was included in our study. We searched the whole, intact Bos d 9 sequence (UniProt P02662) for DRB1*15:01 binders using EpiTOP, and found 5 high binders ( $\mathrm{pIC}_{50}>6.3$; data not shown). However, after the in silico digestion of Bos d 9, no DRB1*15: 01 binders remained. Savilahti et al. [19] found that the DR15-DQB1*06:02 haplotype is associated with high levels of Bos d 5-specific- and Bos d 9/Bos d 10-specific total
IgG and IgG4 in children with CMA, but not among control subjects. In the same study, the DR1/10-DQB1*05:01 haplotype was associated with lower levels of Bos d 5-specific and Gal d 2-specific total IgG and IgG4, particularly in control subjects. In this study, no binders from Bos $\mathrm{d} 5$ were found to DR15 or DQ5. The duodenal enzymes trypsin and chymotrypsin completely degraded the protein in $30 \mathrm{~min}$ [30]. The only peptide that survived after the in silico digestion was part of a known $\mathrm{T}$ cell epitope, but no binders to any of the DRB1 and DQ alleles considered in this study were found (online suppl. table 3 ). In good agreement with the clinical studies, we found 12 binders to DQ6 originating from Bos d 9 (average $\mathrm{pIC}_{50}=6.889$; online suppl. table 5). However, Gal d 2 was found to have 5 strong binders to DR1 (average $\mathrm{pIC}_{50}=$ 7.807) and 7 intermediate binders to DQ5 (average $\mathrm{pIC}_{50}=6.655$ ). The lower allergenicity of Gal $\mathrm{d} 2$ that was found experimentally could be explained by its sensitivity to heat denaturation, a process that was not considered in this study.

\section{Disclosure Statement}

The authors are members of FA COST Action FA1402: Improving Allergy Risk Assessment Strategy (ImpARAS) for new food proteins.

\section{References}

1 Robinson J, Halliwell JA, McWilliam H, Lopez R, Parham P, Marsh SGE: The IMGT/ HLA Database. Nucl Acids Res 2013; 41:D1222-D1227.

2 Fernández MM, Guan R, Swaminathan CP, Malchiodi EL, Mariuzza RA: Crystal structure of staphylococcal enterotoxin I (SEI) in complex with a human major histocompatibility complex class II molecule. J Biol Chem 2006; 281:25356-25364.

3 Kim CY, Quarsten H, Bergseng E, Khosla C, Sollid LM: Structural basis for HLA-DQ2mediated presentation of gluten epitopes in celiac disease. Proc Natl Acad Sci 2004;101: 4175-4179.

4 Dai S, Murphy GA, Crawford F, Mack DG, Falta MT, Marrack P, Kappler JW, Fontenot AP: Crystal structure of HLA-DP2 and implications for chronic beryllium disease. Proc Natl Acad Sci 2010;107:7425-7430.

5 Knapp B, Fisher G, van Hemelen D, Fae I, Maillere B, Edner C, Schreiner W, Bohle B, Jahn-Schmid B: Association of HLA-DR1 with the allergic response to the major mugwort pollen allergen: molecular background. BMC Immunol 2012;13:43.
6 Rajagopalan G, Tilahun AY, Iijima K, David CS, Kita H, Juhn YJ: HLA-DR polymorphism modulates response to house dust mites in a transgenic mouse model of airway inflammation. Tissue Antigens 2011;77: 589-592.

7 Ivkovic-Jurekovic I, Zunec R, Balog V, Grubic Z: The distribution of HLA alleles among children with atopic asthma in Croatia. Coll Antropol 2011;35:1243-1249.

8 Dzurilla M, Vrlik M, Homolova M, Buc M: No association between bronchial asthma and HLA-DRB1 - DQB1 alleles in the Slovak population. Bratisl Lek Listy 2013;114:9395.

9 Kusano S, Kukimoto-Niino M, Satta Y, Ohsawa N, Uchikubo-Kamo T, Wakiyama M, Ikeda M, Terada T, Yamamoto K, Nishimura Y, Shirouzu M, Sasazuki T, Yokoyama S: Structural basis for the specific recognition of the major antigenic peptide from the Japanese cedar pollen allergen Cry $\mathrm{j} 1$ by HLADP5. J Mol Biol 2014;426:3016-3027.
10 Kauppinen A, Perasaari J, Taivainen A, Kinnunen T, Saarelainen S, Rytkonen-Nissinen $\mathrm{M}$, Jeal $\mathrm{H}$, Jones $\mathrm{M}$, Virtanen $\mathrm{T}$ : Association of HLA class II alleles with sensitization to cow dander Bos d 2, an important occupational allergen. Immunobiology 2012;217:812.

11 Jahn-Schmid B, Pickl WF, Bohle B: Interaction of allergens, major histocompatibility complex molecules and T-cell receptors: a 'ménage à trois' that opens new avenues for therapeutic intervention in type I allergy. Int Arch Allergy Immunol 2011;156:27-42.

12 Hourihane JO: Peanut allergy. Pediatr Clin North Am 2011;58:445-458.

13 Shreffler WG, Cherlop-Powers Z, Sicherer SH: Lack of association of HLA class II alleles with peanut allergy. Ann Allergy Asthma Immunol 2006;96:865-869.

14 Dreskin SC, Tripputi MT, Aubrey MT, Mustafa SS, Atkins D, Leo HL, Song B, Schlichting D, Talwar H, Wang Q, Freed BW: Peanut-allergic subjects and their peanut-tolerant siblings have large difference in peanut-specific IgG that are independent of HLA class II. Clin Immunol 2010;137:366-373. 
15 Madore A, Vaillantcourt VT, Asai Y, Alizadehfar R, Ben-Shoshan M, Michel DL, Kozyrskyj AK, Becker A, Chan-Yeung M, Clarke AE, Hull P, Daley D, Sandford AJ, Laprise C: HLA-DQB1*02 and DQB1*06:03P are associated with peanut allergy. Eur J Hum Genet 2013;21:1181-1184.

16 Hong X, Hao K, Ladd-Acosta C, Hansen KD, Tsai HJ, Liu X, Xu X, Thornton TA, Caruso D, Keet CA, Sun Y, Wang G, Luo W, Kumar R, Fuleihan R, Singh AM, Kim JS, Story RE, Gupta RS, Gao P, Chen Z, Walker SO, Bartell TR, Beaty TH, Fallin MD, Schleimer R, Holt PG, Nadeau KC, Wood RA, Pongracic JA, Weeks DE, Wang X: Genome-wide association study identifies peanut allergy-specific loci and evidence of epigenetic mediation in US children. Nat Commun 2015;6:6304.

17 Pascal M, Konstantinou GN, Masilamani M, Lieberman J, Sampson HA: In silico prediction of Ara h $2 \mathrm{~T}$ cell epitopes in peanut-allergic children. Clin Exp Allergy 2012;43:116127.

18 Ruiter B, Rozemuller EH, van Dijk AJ, Garssen J, Bruijnzeel-Koomen CAFM, Tilanus MG, Knol EF, van Hoffen E: Role of human leucocyte antigen DQ in the presentation of $\mathrm{T}$ cell epitopes in the major cow's milk allergen as1-casein. Int Arch Allergy Immunol 2007; 143:119-126.

19 Savilahti EM, Ilonen J, Kiviniemi M, Saarinen KM, Vaarala O, Savilahti E: Human leukocyte antigen (DR1)-DQB1*0501 and (DR15)DQB1*0602 haplotypes are associated with humoral responses to early food allergens in children. Int Arch Allergy Immunol 2010; 154:169-177.

20 Helmberg W, Dunivin R, Feolo M: The sequencing-based typing tool of dbMHC: typing highly polymorphic gene sequences. Nucleic Acids Res 2005;32:W173-W175.

21 Mari A, Rasi C, Palazzo P, Scala E: Allergen databases: current status and perspectives. Curr Allergy Asthma Rep 2009;9:376-383.

22 The UniProt Consortium: UniProt: a hub for protein information. Nucleic Acids Res 2015; 43:D204-D212.
23 Bu G, Luo Y, Chen F, Liu K, Zhu T: Milk processing as a tool to reduce cow's milk allergenicity: a mini-review. Dairy Sci Technol 2013; 93:211-223.

24 Verhoeckx KCM, Vissers YM, Baumert JL, Faludi R, Feys M, Flanagan S, HerouetGuicheney C, Holzhauser T, Shimojo R, van der Bolt N, Wichers H, Kimber I: Food processing and allergenicity. Food Chem Toxicol 2015;80:223-240.

25 Gasteiger E, Hoogland C, Gattiker A, Duvaud S, Wilkins MR, Appel RD, Bairoch A: Protein identification and analysis tools on the ExPASy server; in Walker JM (ed): The Proteomics Protocols Handbook. Totowa, Humana Press Inc., 2005, pp 571-607.

26 Dimitrov I, Garnev P, Flower DR, Doytchinova I: EpiTOP - a proteochemometric tool for MHC class II binding prediction. Bioinformatics 2010;26:2066-2068.

27 Dimitrov I, Doytchinova I: Peptide binding to five most frequent HLA-DQ proteins - a proteochemometric approach. Mol Informatics 2015;34:467-476.

28 Astwood JD, Leach JN, Fuchs RL: Stability of food allergens to digestion in vitro. Nature Biotechnol 1996;14:1269-1273.

29 Creamer LK, Loveday SM, Sawyer L: Beta-lactoglobulin; in Fuquay JW, Fox PF, McSweeney PLH (eds): Encyclopedia of Dairy Sciences. San Diego, Academic Press, 2011, vol 3, pp 787-794.

30 Benedé S, López-Expósito I, Giménez G, Grishina G, Bardina L, Sampson HA, LópezFandiňo R, Molina E: Mapping of IgE epitopes in in vitro gastroduodenal digests of $\beta$-lactoglobulin produced with human and simulated fluids. Food Res Int 2014;62:11271133.

31 Tanabe S, Kobayashi Y, Takahata Y, Morimatsu F, Shibata R, Nishimura T: Some human $B$ and $T$ cell epitopes of bovine serum albumin, the major beef allergen. Biochem Biophys Res Commun 2002;293:1348-1353.
32 Docena GH, Fernandez R, Chirdo FG, Fossati CA: Identification of casein as the major allergenic and antigenic protein of cow's milk. Allergy 1996;51:412-416.

33 Bernard H, Créminon C, Yvon M, Wal JM: Specificity of the human IgE response to the different purified caseins in allergy to cow's milk proteins. Int Arch Allergy Immunol 1998;115:235-244.

34 Lisson M, Lochnit G, Erhardt G: In vitro gastrointestinal digestion of bovine as1- and as2casein variants gives rise to different IgEbinding epitopes. Int Dairy J 2014;34:47-55.

35 Honma K, Aoyagi M, Saito K, Nishimura T, Sugimoto K, Tsunoo H, Niimi H, Kohno Y: Antigenic determinants on ovalbumin and ovomucoid: comparison of the specificity of IgG and IgE antibodies. Jpn J Allergol 1991; 40:1167-1175.

36 Matsuda T, Watanabe K, Nakamura R: Immunochemical and physical properties of peptic-digested ovomucoid. J Agric Food Chem 1983;31:942:946.

37 Bernhisel-Broadbent J, Dintzis HM, Dinztis RZ, Sampson HA: Allergenicity and antigenicity of chicken egg ovomucoid (Gal d III) compared with ovalbumin (Gal d I) in children with egg allergy and in mice. J Allergy Clin Immunol 1994;95:1047-1059.

38 Caubet JC, Wang J: Current understanding of egg allergy. Pediatr Clin North Am 2011;58: 427-443.

39 Quirce S, Maranon F, Umpierrez A, de las Heras M, Fernandez-Caldas E, Sastre J: Chicken serum albumin (Gal d 5) is a partially heat-labile inhalant and food allergen implicated in the bird-egg syndrome. Allergy 2001;56:754-762.

40 Dhanapala P, De Silva C, Doran T, Suphioglu C: Cracking the egg: an insight into egg hypersensitivity. Mol Immunol 2015;66:375-383.

41 Amo A, Rodriguez-Perez R, Blanco J, Villota J, Juste S, Mone I, Caballero ML: Gal d 6 is the second allergen characterized from egg yolk. J Agric Food Chem 2010;58:7453-7457. 